\title{
DISGRESIONES SOBRE LA CRONOLOGIA DEL ARTE RUPESTRE «LEVANTINO»
}

\author{
ANTONIO BELTRÁN
}

Queremos asociarnos al homenaje al Prof. Nieto Gallo con estas breves notas, sin aparato erudito, planteadas como digresiones que bien podrían apuntar a una revisión del estado de la cuestión en tema tan debatido como el de la cronología del arte llamado, convencionalmente, levantino, cuyas vicisitudes llevan desde la datación paleolítica postulada por Breuil y seguida por todos los grandes prehistoriadores de su tiempo, a la crítica, esencialmente de españoles de la época de Hernández Pacheco, Martínez Santa-Olalla y M. Almagro, que se inclinaron por una fecha meso-neolítica, unánimemente admitida hasta nuestros días, con la excepción de la tardía datación de F. Jordá que lo incluye, en gran parte, en la Edad del Bronce.

En 1968 nos inclinábamos por un principio de este arte en el sexto milenio a. C. y un final provocado por la extensión del arte "esquemático» de la Edad del Bronce, tras una fase de coexistencia, produciéndose una solución de continuidad entre el final de los llamados polícromos del Magdaleniense VI y el principio del arte naturalista levantino en los animales e impresionista en la figura humana, y una lenta evolución y proceso de estilización en el arte levantino para incorporar ideas nuevas abstractas y esquemáticas supuestas de procedencia oriental y del IV milenio en su origen para alcanzar la Península en la zona sur en el siguiente.

Recientes descubrimientos y trabajos actuales en la propia Península, en el norte de Africa y en el sur de Italia vienen a complicar mucho la aparente sencillez del esquema habitualmente admitido y a negar algunos de los datos que parecían firmes y que sustentaban las hipótesis que se aceptaban sin discusión. Nuestra datación del 6.000 para los inicios del arte levantino y las relaciones que suponíamos, de una forma vaga, con el norte y centro de Africa, fueron duramente discutidas o simplemente ignoradas.

En el momento presente el supuesto "hiatus» entre el naturalismo de las pinturas parietales y los grabados sobre pared o de arte mobiliar del Magdaleniense final y el arte levantino, que parecía absurdo tratar de cubrir con los cantos pintados azilienses o con el geometrismo, por ejemplo, de las placas de la Cocina, es menos claro de lo que parecía; en primer lugar el "estilo magdaleniense» se definía por 
el método comparativo, arrastrando a tal datación figuras que no tenían ninguna posibilidad de ser fechadas independientemente; se olvidaba la observación de Breuil sobre diversas figuras naturalistas pintadas, por ejemplo en Niaux, que incluía en una etapa aziliense. En los últimos años plaquetas grabadas con figuras de estilo que puede calificarse de magdaleniense están apareciendo en niveles azilienses seguros y si comparamos con ellas figuras de arte parietal es posible que haya que rebajar considerablemente el final del arte paleolítico que se hacía terminar bruscamente, precisamente en el momento de su apogeo, por razones climáticas.

Por otra parte en numerosos abrigos levantinos se ha comprobado que las figuras "clásicas" naturalistas o impresionistas cortan otras anteriores geométricas que se asocian en la región de Cocentaina con representaciones antropomórficas de gran tamaño, poco realistas, pero sin dudas sobre su identificación. Esto lo advertimos en la cueva de la Sarga (Alcoy), después en La Araña y en Cantos de la Visera (Yecla), luego en Balsa de Calicanto (Bicorp) por Aparicio y en Labarta, en la provincia de Huesca, por Baldellou y de nuevo por nosotros en dos abrigos del barranco de la Valltorta y en los Chaparros de Albalate del Arzobispo; es decir, se trata de un fenómeno general que tiene el mayor número de ejemplos en la zona de Murcia y Alicante, pero que, sin duda, se encontrará en todas partes cuando se busque adecuadamente.

Quiere decirse que independientemente de la fecha que se asigne a las más antiguas pinturas "levantinas" clásicas, hay una fase anterior y estilísticamente completamente diferente con fuerte tendencia al simbolismo respecto de las figuraciones y al geometrismo en la expresión habitual que, al menos en los casos citados, fue pintada en los mismos lugares que luego ocuparon con su arte los «levantinos", en tanto que el arte paleolítico se prolongaba durante el aziliense, acercando mucho los dos extremos cronológicos del "hiatus" inexplicado e inexplicable, sobre todo cuando el abate Breuil enlazaba el estilo de las grandes figuras estáticas de animales levantinos con las tintas planas de su ciclo auriñaco-perigordiense.

Claro que lo dicho sirve para criticar objetivamente las hipótesis vigentes, pero carece de datos y, por lo tanto, de fuerza suficiente para proponer otra conclusión general que no sea la provisional de que entre el Magdaleniense final y el inicio del arte Levantino existe una fase artística intermedia que hasta ahora no puede datarse con exactitud y que no supone una transición estilística entre uno y otro artes $e$ incluso puede asegurarse que se separa, artísticamente, de ellos manifestándose solamente en la zona luego se desarrollará el arte levantino.

Por otra parte siempre ha constituido un misterio el estímulo que provocó el nacimiento del arte levantino si no continuaba la tradición paleolítica. Lo que sabíamos del arte norteafricano con una supuesta datación postneolítica y las dificultades que se oponían a la travesía mediterránea hacían que las escasas semejanzas formales del arte del Tassili n'Ajjer y el levantino fueran rechazadas de plano. Los estudios en curso sobre el fenómeno de desecación y subsiguiente desertización del norte de Africa han permitido conocer que tras milenios de sequedad hacia el 10.000 a. C. se produjo una fase de humedad que provocó la vuelta del poblamiento humano y animal con una intensa actividad que cambió de nuevo cuando hacia el 5.000 ó 6.000 B.P. el desierto comenzó a recuperar las tierras que habían conocido 
varios milenios de florecimiento a juzgar por grabados y pinturas obligando a las bestias y a los hombres a emigrar en un proceso que continúa aún hoy con signos alarmantes. Por otra parte F. Mori ha postulado unas fechas para el arte sahariano que aunque no sean aceptadas por todos nos parecen justas y que resumimos de la forma siguiente (todas B.P., desde hoy: 1) Fase del búbalo, de los animales salvajes o de los cazadores, 8000 con granos de mijo y 7045 asociada a colores procedentes de las paredes pintadas, todas en estaciones del Tadrart Acacus, en la actual Libia. 2) Fase de las cabezas redondas, con fecha ante quem del 6754 y otras hasta 5405 sobre frontal de dos brachyceros y momia infantil; 3) Fase pastoril, con 4730 ante quem para la fase media y seriación de la fase reciente con figuras pintadas en los vacíos dejados al caer la superficie sobre la que se pintó en la fase anterior. Las fases posteriores del caballo con carro o sin él y del camello no interesan a nuestros fines.

En el estado actual de nuestros conocimientos sería atrevido, aunque sugestivo, comparar la fase de cabezas redondas comprendiendo grandes personajes fantásticos, de grandes cabezas, con las de dimensiones semejantes del arte "prelevantino" de Castell de Castells, en el Pla de Petracos y otras localidades de la zona de Cocentaina. Y mucho más pensar que estos fantásticos personajes con cabezas radiadas y manos de las que brotan también radiaciones, puedan ser el origen de las figuras seminaturalistas o esquemáticas que aparecen en negro o en rojo, respectivamente, en los Estrechos de Albalate del Arzobispo puestos en pie sobre bóvidos y en un caso, hombre y mujer. La introducción del caballo en el Sahara hacia el 1500 a. C. podría muy bien relacionarse con el jinete con casco y riendas del barranco de Gasulla.

No hay posibilidad de establecer comparaciones directas del arte sahariano con el levantino español, pero resulta al menos curioso que ambos se desarrollen a lo largo del mismo período cronológico y con alternativas climáticas que pueden ser comunes y que, en el peor de los casos, motivaron trágicos movimientos de las poblaciones norteafricanas, bien documentadas hacia el Níger e ignoradas en cualquiera otra dirección. La secuencia Epipaleolítico-Neolítico está muy clara en el esquema de Mori.

En lo que se refiere al momento final del arte «levantino" no cabe duda que a la evolución producida por su dinámica interna que ha podido seguirse en lo que se refiere a las formas de hombres y animales, colores, movimiento, tamaño y estilización hay que sumar los cambios culturales que se introducen con las nuevas ideas de los metalúrgicos, expresiones plásticas geométricas, abstractas y simbólicas hasta llegar al esquematismo. Desde el excelente trabajo de Pilar Acosta que planteaba la arribada desde el Oriente próximo de esquemas nacidos en el siglo IV y su aculturación en la Península a lo largo del milenio siguiente, el problema radicaba en analizar de qué forma se fundían los elementos llegados del exterior con los evolucionados del arte levantino en la zona donde éste se hallaba presente y la medida en que pudo influir en las expresiones que llegarían alrededor del megalitismo y los nuevos modos de enterramiento y de los cambios en la vida social y económica. Para quienes pensábamos que el arte esquemático no era, en lo esen- 
cial, una consecuencia de la evolución del levantino, dado que se extendía por toda la Península y en territorios donde no existía precedente alguno levantino, el descubrimiento de las dos cuevas de la zona de Otranto, sobre todo Porto Badisco, pero también en Cosma, venían a aportar novedades absolutamente revolucionarias. A este descubrimiento se unía el descubrimiento de las cuevas de la Peñarrubia de Cehegín con figuras rojas que un pintor de la localidad dijo haber pintado él y que sometidas a nuestra opinión nos parecen absolutamente auténticas, con torpes y desmañadas pinturas rojas que en una de las tres cuevas se hallaban en pared situada sobre enterramientos eneolíticos y cuyos cuerpos humanos prolongados para formar largas cabezas, brazos doblados y actitudes curvadas se repitieron en la única figura humana visible con claridad de la cueva de la Higuera en Cartagena y en uno de los grupos de Porto Badisco. En nuestro trabajo inédito sobre las cuevas de las Palomas, las Conchas y el Humo (realmente éstas dos parte de una sola) hacemos notar la torpeza de realización y lo extraño de las formas de estas figuras y pensábamos en una zona perimetral, en Cehegín, en donde el estilo rotundamente levantino y de ninguna forma esquemático o evolucionado en esta dirección, habría podido producirse por dinámica interna en círculo cerrado, quizá en una etapa cronológica avanzada, pero sin la menor conexión con los hombres en "phi" o los cuadrúpedos esquemáticos, por ejemplo del abrigo de El Pozo de Calasparra.

Así las cosas y en curso aún de estudio estos problemas, el análisis de visu y detenido que hemos podido hacer de Porto Badisco en el verano de 1986 nos permite esbozar hipótesis de trabajo que, de ser válidas, harían cambiar no poco las mantenidas hasta ahora.

Aunque la cueva fue descubierta en 1970 y publicada por P. Graziosi diez años más tarde, las excavaciones anuales continúan y las dataciones se establecen cada vez con mayor seguridad. La cueva se cerró en el Eneolítico lo que garantiza una fecha anterior y en el interior se han hallado cerámicas y "pintaderas" que reproducen los temas pintados en las paredes, con dataciones de hasta el V milenio para figuras que tipológicamente se clasificarían entre las muy evolucionadas. A la idea de que la zona de Otranto fuese una escala en el camino entre el Oriente Próximo y el Sudeste de la Península, en el que podría incluirse también Olmetta-du-Cap en Córcega y a la identificación de algunas formas esquemáticas, especialmente la "diosa de los ojos" de Tell Brak, viene a perturbar su buen sentido la considerable antigüedad de las figuras de Porto Badisco. La cueva tuvo tres espacios o ambientes con pinturas y muros construidos en el interior donde las cerámicas más antiguas van a una fase muy primitiva del neolítico de Serra d'Alto. El conjunto más antiguo es de color rojo y todas las figuras se agrupan en la primera sala de la caverna. La semejanza con las del interior de cueva de Peñarrubia es verdaderamente asombrosa, tanto en las formas como en el color rojo claro y vivo obtenido partiendo del óxido férrico; las pinturas negras están realizadas con guano de murciélago. Volviendo al conjunto rojo presenta junto a animales esquemáticos, la casi totalidad cuadrúpedos, hombres estilizados, pero más realistas con los brazos curvados y las cabezas formadas por una prolongación del cuerpo. Hay que advertir 
que los tosquísimos animales de Peñarrubia nada tienen que ver con los de Porto Badisco, uno de ellos un cérvido tosquísimo, pero naturalista con las emplumaduras de tres flechas visibles claramente. Una figura roja de una cerámica de Porto Badisco anterior al 4000 reproduce una de las parietales y fue hallado junto a ella. Las estilizaciones humanas llegan a un grado extremo en las pinturas negras que repiten temas de "pintaderas" neolíticas y de cerámicas como una del Instituto de Paletnología de Florencia, idéntico a una de las formas simbólicas llamadas "complejas" o "colectivas" por Graziosi en otros casos. Dejando aparte su interpretación como figuras humanas la indicación de ritos parece clara; una figura en forma de cruz de Malta está sobre un arco natural que comunica dos salas con pinturas y en la de más al fondo varias decenas de manitas infantiles forman un inquietante conjunto inexplicable. Las indiscutibles relaciones con la cueva Magura o Magurata de Bulgaria, o las citadas españolas se añaden a la corriente de pintaderas que desde Tesalia pueden llegar hasta la Isla de Gran Canaria con una sorprendente semejanza de formas. En el Museo de Tarento se conserva una pintadera de la cueva de l'Erba di Avetrana con uno de los temas de las pinturas parietales de Porto Badisco.

La cueva de Cosma, hoy inaccesible, con una decena de figuras, repite los problemas de Porto Badisco, muy cerca de la cual se abre.

En resumen, la datación de la mayor parte de las pinturas entre los milenios IV y III y la posibilidad de que las figuras rojas lleguen hasta el V descompone la lógica marcha Oriente-Occidente a partir de los ejemplos bien datados y deja al extraordinario conjunto hispánico de "arte esquemático» en tiempos bastante posteriores. Lo verdaderamente importante es pensar que si Porto Badisco permite llevar sus figuras más antiguas a una fecha semejante a las más naturalistas, estáticas y de tintas planas muy patinadas, del Levante español habría que pensar en qué medida Peñarrubia y la Higuera podrían ser una consecuencia de aquéllas y no corresponder a una evolución de las levantinas clásicas.

Por otra parte el descubrimiento aún inédito de Los Estrechos de Albalate del Arzobispo (Teruel) viene a poner de manifiesto, de nuevo, la cuestión del arte "subnaturalista» que ya planteó Bosch Gimpera, ni levantino ni esquemático, y que sale de los esquemas monolíticos generales. En efecto, en dicho abrigo, colgado sobre el río Martín y a más de cuarenta metros de altura y hoy totalmente inaccesible, existen figuras esquemáticas rojas, entre las que interesa especialmente un ancoriforme humano sobre un cuadrúpedo en rojo, puesto en pie; otras figuras rojas también esquemáticas cortan a figuras negras seminaturalistas, de fuerte tendencia realista en los animales, especialmente en un equino y un bovino y sobre éste, y también en pie, una pareja de hombre y mujer, ambos con las cabezas radiadas y los dedos de manos y pies radiantes y la figura femenina con ostentación de una vulva de gran tamaño formada por un óvalo con una línea vertical. La cronología relativa de colores que da mayor antigüedad al rojo sobre el negro aquí se invierte sin la menor duda y las figuras negras de animales que no son de estilo levantino, mucho menos pueden ser calificadas de esquemáticas. Esto no es ninguna novedad, pero significa una evolución especial que no encaja en la admitida para el arte levantino. Muy cerca de Los Estrechos, en el mismo cañón y a su entrada, hay un abrigo 
levantino con figuras clásicas, entre ellas una superposición de dos arqueros cazando un jabalí, cortando pinturas geométricas "prelevantinas" y otras figuras de hombres y animales que podríamos llamar clásicas; hay también figuras subnaturalistas en

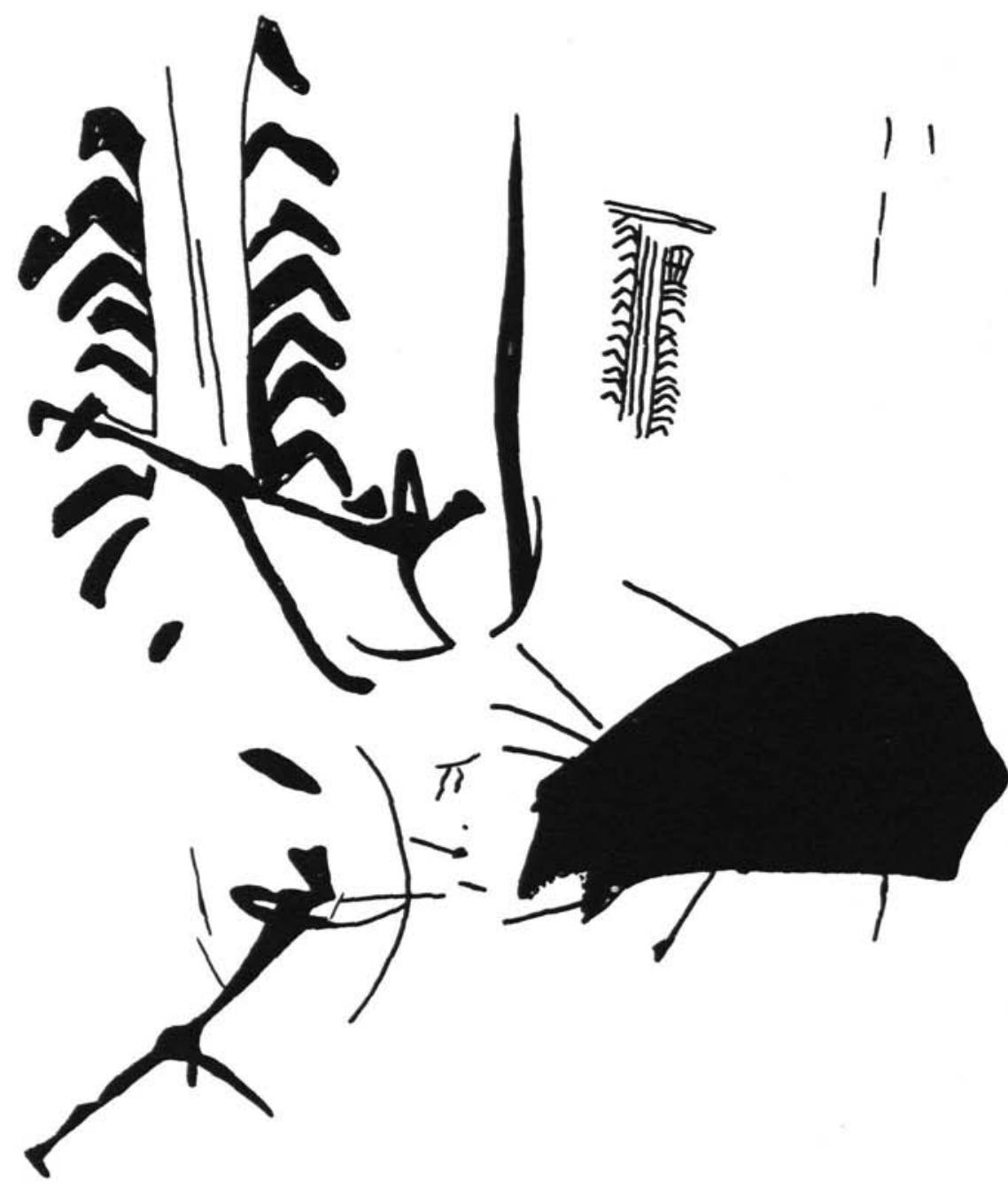

Los Chaparros (Albalate del Arzobispo, Teruel). Arqueros asaltando un jabalí sobre pinturas geométricas prelevantinas (Yacimiento inédito según A. Beltrán). 
rojo y algunas esquemáticas, pero ninguna que pueda encajar en una secuencia que incluya las figuras seminaturalistas y esquemáticas del vecino yacimiento de Los Estrechos, como si se tratase de dos artes distintos, ambos sucediéndose en cada lugar, pero independientemente y con arreglo a normas totalmente separadas.

Estas disgresiones pretenden mostrar que quizá hemos fiado excesivamente en hipótesis de trabajo que los nuevos descubrimientos vienen a discutir. Si el arte levantino propio de cazadores de serranía aparece ahora a la orilla del mar y si a su decantada consubstancialidad con la luz y el aire libre hay que oponer su aparición en el interior de las cuevas de Peñarrubia, de las Arañas del Carabasí de Santa Pola o de Sant Esteve de les Gralles en Lérida, habría que añadir, también hipotéticamente, en primer lugar, una ampliación de los términos cronológicos, con una fase "prelevantina" seguramente muy anterior al 6000 y fundamentalmente, la aceptación de que no se trata de un arte sometido a leyes fijas y únicas de evolución, que podría relacionarse con análogos procesos en la zona sahariana y con muy antiguas manifestaciones emparentadas con él en Bulgaria y el sur de Italia, con lo que hasta ahora conocemos. Finalmente, a las muchas críticas que el "orientalismo» de las manifestaciones del Neolítico y la Edad del Bronce en la Península está recibiendo, puede sumarse la que resulte de la gran antigüedad de las pinturas de Porto Badisco, que están muy lejos de ser un fenómeno aislado y excepcional. Por otra parte no conocemos aún el alcance del descubrimiento de las pinturas paleolíticas de la cueva Fosca de la Vall d'Ebo, en Cocentaina, cerca del conjunto "prelevantino" y "levantino" tantas veces aludido y aún no se han incorporado a los esquemas cronológicos generales del arte paleolítico las dataciones de hasta el 40.000 en yacimientos pintados de Tanzania y muy antiguos en otros lugares de Africa, que están acosando con severas dudas a las grandes construcciones teóricas sobre el arte cuaternario. Quizá en poco tiempo se demostrará que, como suele ocurrir, los fenómenos de la cultura humana son más complejos y difíciles de explicar que lo simplificado y sintetizado por las teorías que sobre ellos construimos.

La justificación de las opiniones vertidas en esta nota pueden verse en: Antonio Beltrán, "L'art préhistorique espagnol: Nouveaux horizons et problemes: Etat de la question", en prensa en Bolletino del Centro Camuno di Studi Preistorici, 1987. Varios, Arte Preistorico del Sahara, Firenze, 1986. Fabrizio Mori, Tadrart Acacus. Arte rupestre e culture del Sahara preistorico, Torino, 1965. Emmanuel Anati, "Magourata Cave», Bolletino del Centro Camuno, cit. IV, 1971 y "The state of Research in Rock Art: The Rock Art of Tanzania and the East African Sequencen, Ibidem, XXIII, pág. 15, 1986. P. Graziosi, Le pitture preistoriche della grotta di Porto Badisco, Firenze, 1980. A. Beltrán, "Las pinturas de Porto Badisco y el arte parietal esquemático español", Annali del Museo Civico U. Formentini della Spezia, 1979-80, La Spezia, 1982. A. Beltrán, «El arte prelevantino", en prensa en Homenaje a D. Fletcher, S.I.P., Valencia y Las pinturas rupestres de la Peña Rubia, Cehegín, Murcia y el problema de su autenticidad, Informe al Ministerio de Cultura, abril 1984 y con M. San Nicolás, "Las pinturas rupestres de la Peña Rubia de Cehegín, Murcian, Revista de Arqueología, VI, Madrid, 1985, pág. 9. La bibliografía complementaria en las obras citadas y en A. Beltrán, Da cacciatori ad allevatori. L'arte rupestre del Levante spagnolo, Milano, 1982. 